\title{
Is there an upper limit of intracranial pressure in patients with severe head injury if cerebral perfusion pressure is maintained?
}

\author{
Jeffrey S. Young, M.D., Osbert Blow, M.D., Ph.D., \\ Florence Turrentine, R.N., B.S.N., JeFFrey A. Claridge, M.D., \\ AND ANDREW Schulman, M.D.
}

University of Virginia Trauma Center, University of Virginia Health System, Charlottesville, Virginia

\begin{abstract}
Authors of recent studies have championed the importance of maintaining cerebral perfusion pressure (CPP) to prevent secondary brain injury following traumatic head injury. Data from these studies have provided little information regarding outcome following severe head injury in patients with an intracranial pressure (ICP) greater than $40 \mathrm{~mm} \mathrm{Hg}$, however, in July 1997 the authors instituted a protocol for the management of severe head injury in patients with a Glasgow Coma Scale score lower than 9. The protocol was focused on resuscitation from acidosis, maintenance of a $\mathrm{CPP}$ greater than $60 \mathrm{~mm} \mathrm{Hg}$ through whatever means necessary as well as elevation of the head of the bed, mannitol infusion, and ventriculostomy with cerebrospinal fluid drainage for control of ICP. Since the institution of this protocol, nine patients had a sustained ICP greater than $40 \mathrm{~mm} \mathrm{Hg}$ for 2 or more hours, and five of these had an ICP greater than $75 \mathrm{~mm} \mathrm{Hg}$ on insertion of the ICP monitor and later experienced herniation and expired within 24 hours. Because of the severe nature of the injuries demonstrated on computerized tomography scans and their physical examinations, these patients were not aggressively treated under this protocol. The authors vigorously attempted to maintain a CPP greater than $60 \mathrm{~mm} \mathrm{Hg}$ with intensive fluid resuscitation and the administration of pressor agents in the four remaining patients who had developed an ICP higher than $40 \mathrm{~mm} \mathrm{Hg}$ after placement of the ICP monitor. Two patients had an episodic ICP greater than $40 \mathrm{~mm} \mathrm{Hg}$ for more than 36 hours, the third patient had an episodic ICP greater than of $50 \mathrm{~mm} \mathrm{Hg}$ for more than 36 hours, and the fourth patient had an episodic ICP greater than $50 \mathrm{~mm} \mathrm{Hg}$ for more than 48 hours. On discharge, all four patients were able to perform normal activities of daily living with minimal assistance and experience ongoing improvement.

Data from this preliminary study indicate that intense, aggressive management of CPP can lead to good neurological outcomes despite extremely high ICP. Aggressive CPP therapy should be performed and maintained even though apparently lethal ICP levels may be present. Further study is needed to support these encouraging results.
\end{abstract}

KeY WORDS - head injury - cerebral perfusion pressure • trauma

The current management of neurological trauma has undergone great change in the past 5 years. Initial studies by Becker, et al., ${ }^{2}$ featured a coherent strategy for diagnosing and treating intracranial hypertension, including early diagnosis of intracranial mass lesions, monitoring of parenchymal or intraventricular pressure, elevation of the head of the bed, restriction of fluids, and administration of osmotic diuretic agents. Early data from the TCDB confirmed that an intracranial ICP greater than $20 \mathrm{~mm} \mathrm{Hg}$ for

Abbreviations used in this paper: ARDS = adult respiratory distress syndrome; $\mathrm{CPP}=$ cerebral perfusion pressure; $\mathrm{CT}=$ computerized tomography; GCS = Glasgow Coma Scale; GOS = Glasgow Outcome Scale; ICP = intracranial pressure; ICU = intensive care unit; ISS = injury severity score; MVA = motor vehicle accident; $\mathrm{TBI}=$ traumatic brain injury; $\mathrm{TCDB}=$ Traumatic Coma Data Bank; $\mathrm{UVA}=$ University of Virginia. sustained intervals was associated with poor neurological outcome. ${ }^{11}$ This information led to the acceptance of the aforementioned measures as the mainstays of treatment. Muizelaar and colleagues ${ }^{13}$ have challenged the use of prophylactic hyperventilation therapy in treating acute head injury, although its adverse effects have been accepted by many centers. The greatest controversy concerns the treatment of blood pressure and volume status. Young and Meredith $^{20}$ found that in patients with multiple traumas, a marked positive fluid balance associated with oxygen delivery-directed resuscitation had no adverse effects on neurological outcome or ICP. Rosner and colleagues ${ }^{18,19}$ have convincingly demonstrated that a protocol focusing on the maintenance of CPP in excess of $65 \mathrm{~mm} \mathrm{Hg}$ can improve neurological outcome and patient survival. Even in these studies, however, it is unclear what ICP can be tolerated if CPP is maintained and what the effects on 
patient outcome are in the event of severe, sustained intracranial hypertension (ICP > $30 \mathrm{~mm} \mathrm{Hg}$ ).

Using the guidelines created by the American Association of Neurological Surgeons, we implemented a protocol for severe closed head injury that was focused on adequate total resuscitation, ICP monitoring, and maintenance of a CPP greater than $65 \mathrm{~mm} \mathrm{Hg}$ (Fig. 1). We report our early experience with this treatment strategy.

\section{CLINICAL MATERIAL AND METHODS}

The UVA Trauma Center is a Level I institution serving a referral population of 800,000 people in central Virginia. The trauma team, in conjunction with personnel from the neurosurgical service, initially evaluates all cases of severe head injury. Isolated head trauma is usually managed exclusively by the neurosurgeons, whereas headinjured patients with multiple traumas are treated by the trauma team together with neurosurgical consultation. Initial treatment of severe head injury (GCS Score 8 or less) in the Emergency Department includes early intubation, fluid resuscitation, rapid cranial CT studies, and immedi- ate assessment and treatment of life-threatening injuries. Hyperventilation and mannitol therapies are used in the Emergency Department only if there are clinical signs of cerebral herniation or if the CT scan reveals significant midline shift. If no intracranial lesion requiring surgery exists and there are no other indications for immediate surgery, ICP monitoring is initiated as soon as laboratory tests reveal the absence of a significant coagulopathy (prothrombin time $<15$ seconds). A Codman ICP monitoring system (Johnson and Johnson, Inc., Raynham, MA) is used, and the monitor is placed in the usual fashion in the most injured cerebral hemisphere or in the nondominant hemisphere. According to the policy of the Department of Neurological Surgery at UVA, ventriculostomies are not routinely placed initially. The UVA Medical Center was one of four main institutions participating in the TCDB and the only institution that did not routinely use ventriculostomies. Post hoc analysis of data accumulated in the TCDB revealed no significant difference in outcome in severely head injured patients among participating institutions. Although there have been numerous studies in which investigators examined the relative accuracy for the

Fig. 1. Algorithm for the management of severe head injury accepted by the Neurosurgery and Trauma Services at UVA prior to this study. $\mathrm{CHI}=$ closed-head injury $\mathrm{CI}=$ cardiac index; $\mathrm{CVP}=$ central venous pressure; $\mathrm{D} / \mathrm{C}=$ discontinue; LA = lactic acid; MVO2 sat = mixed venous oxygen saturation; NSGY = neurosurgery; OSM = serum osmolality; PCWP = pulmonary capillary wedge pressure; $\mathrm{SBP}=$ systolic blood pressure; $\mathrm{UO}=$ urine output.

\section{Guidelines for Management of Severe CHI}

UVA Trauma Center

\section{ICP Placement, Sedation, and Monitoring}

\section{ICP Placement}

ICP moniters will be placed at the discretion of the Neurosurgery service. In general, patients wits GCS 9 and/er intracranial mass lesions will require ICP moaitor placemeat.

\section{Sedation}

ICP monitor in place

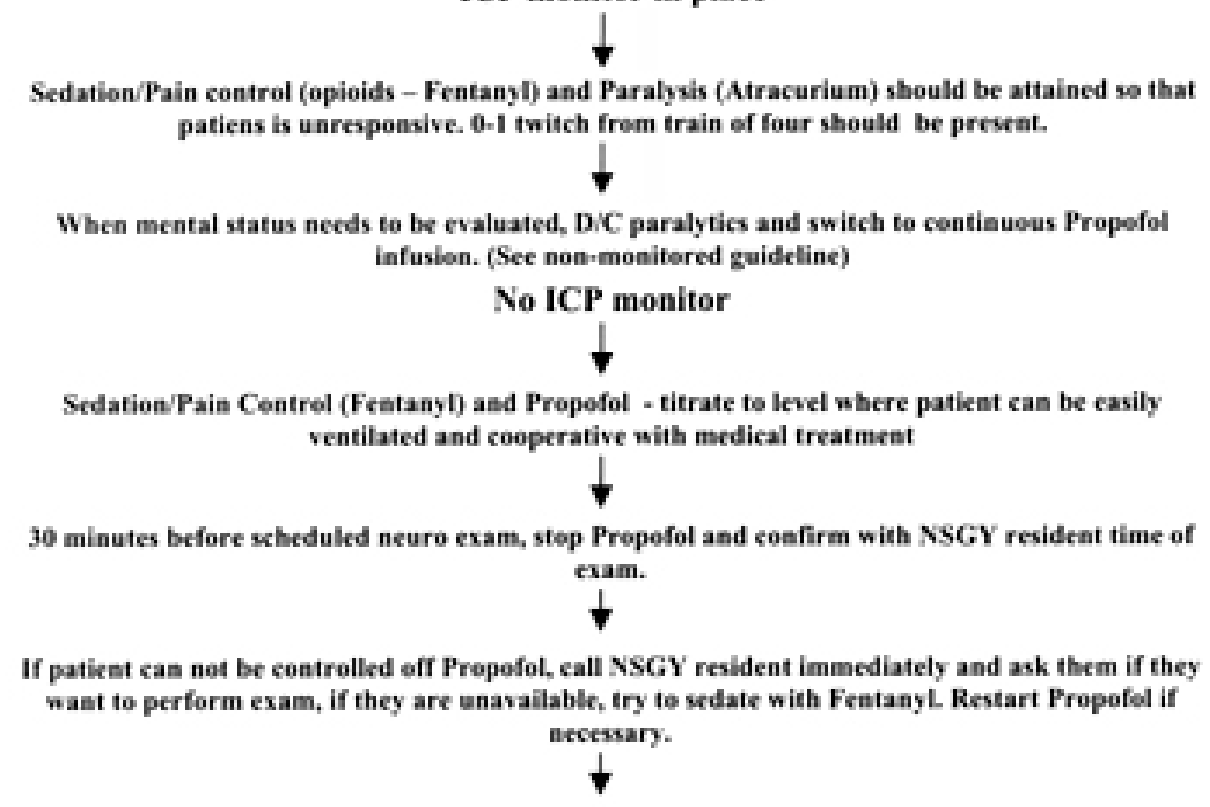

Neuro esam should be performed on NSGY rounds each morning, therefore confirm with NSGY that Propofol can be stopped at 6:30 AM. 


\section{Resuscitation}

\section{Concurrent Resuscitation: (AL.L Paticnts)}

Step blecding, resuscitation with bleod, blood prodects and erystalloid to SBP $>100$, pulse $<100$

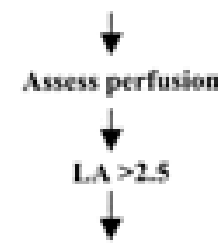

Transfuse fuids to achicve clinically normal perfusion and repeat L.A

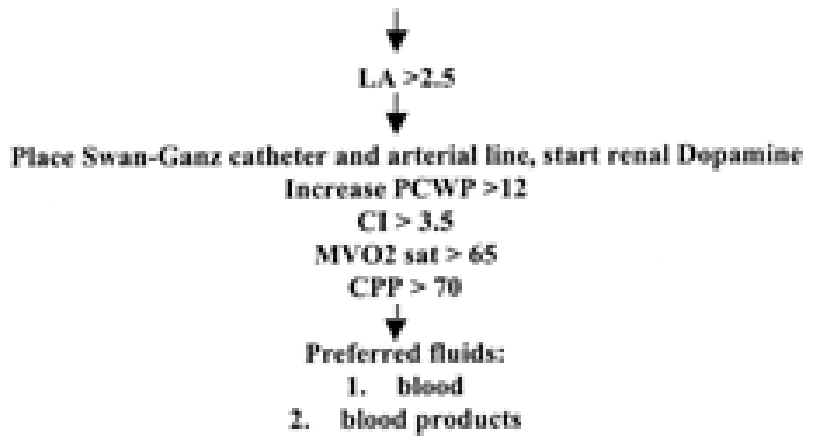

3. albumin or Hespan

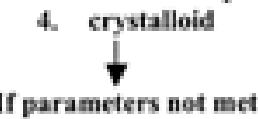

If parameters not met

Add:

Dobetamine

Search for continued bleeding

Goal L $4<25$

\section{Intracranial Hypertension}

Resuscitation Ongoing coupled with Intracranial Hvpertension ( ICP $>25$ )

(Proceed to next step if previous intervention does not bring ICP $<25$ )

Insure adequate sedation and paralysis

Insure CPP $>70$ by above techniques,

insure nermovolemia (CVP at least 8-10, adequate $\mathrm{UO}$, urine $\mathrm{Na}>25$, Serum $\mathrm{OSM}<320$ )

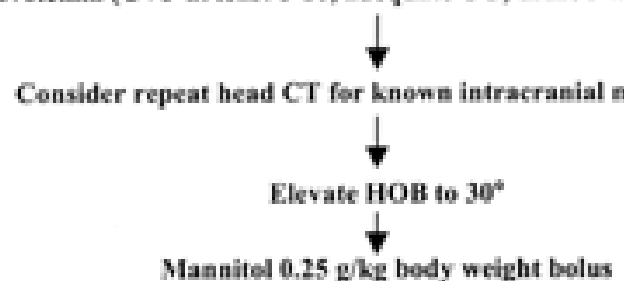

( adequate volume status MUST be present before Mannitol infusion in order to avoid hypotension, renal impairment, and systemie hypoperfusion)

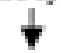

Repeat Mannitol to total of $1 \mathrm{~g} / \mathrm{kg}$ as long as perfusion and volume status maintained

$\checkmark$

Hyperventilate to $\mathrm{pCO} 225-30 \mathrm{~mm} \mathrm{H}$

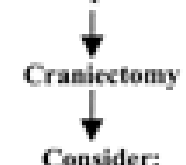

ventricular drainage

phenobarbitel

repeat Head CT 
methods of measuring ICP, no data from any randomized prospective trial in which ventriculostomies were compared with intraparenchymal monitors have demonstrated that ventriculostomy placement and cerebrospinal fluid drainage have a significant benefit for morbidity or mortality rates in similar patients. Thus, these catheters are not part of our standard protocol for the treatment of severe head injury. The protocol for the management of ICP and resuscitation, which was initiated in July 1997, is featured in Fig. 1.

\section{RESULTS}

\section{Patient Population}

Between July 1, 1997, and March 1, 1998, 32 adult patients with blunt injuries and a GCS score of 8 or lower were admitted to the trauma service. Four patients were clinically brain dead on arrival. Eighteen patients underwent placement of ICP monitors and the CPP/ICP treatment protocol was initiated. Nine patients did not have intracranial hypertension and they all survived. Nine patients with a GCS score of 8 or less had a sustained ICP greater than $25 \mathrm{~mm} \mathrm{Hg}$ ( $\geq 2$ hours); five of these patients had a GCS score of 3 on arrival and had evidence of cerebral herniation, but no evidence of an intracranial lesion requiring surgery. Initial ICP measurements were greater than $75 \mathrm{~mm} \mathrm{Hg}$ and these five patients were all declared clinically brain dead within 24 hours; therefore, personnel from the neurosurgery service believed that these patients' conditions were not salvageable and they were not treated according to the protocol. Four patients were treated using the protocol for severe intracranial hypertension.

\section{Illustrative Cases}

Case 1. This 27-year-old woman was struck by a pickup truck. Her condition was stabilized and she was intubated at a referring hospital and then transferred to our center. Her injuries included a severe, open pelvic fracture with massive soft-tissue injury, a closed head injury (GCS Score 3 on admission), an open left tibia-fibula fracture, a scalp laceration, and a small liver laceration. Her initial cranial CT scan demonstrated mild swelling and no intracranial mass lesion. She was hemodynamically stable, with an initial lactic acid level of $9.6 \mathrm{mmol} / \mathrm{L}$. Her ISS ${ }^{1}$ was 50 and her initial ICP was $21 \mathrm{~mm} \mathrm{Hg}$. According to our protocol, she underwent aggressive resuscitation with fluids and pressors in an attempt to correct her lactic acidosis and improve her CPP. The patient experienced a severe decrease in CPP in the 1st few hours after her arrival in the ICU (Fig. 2), which prompted the administration of intravenous pressor agents (Fig. 3). We were able to maintain a CPP greater than $60 \mathrm{~mm} \mathrm{Hg}$ with the aid of significant pressor doses and massive ongoing fluid resuscitation despite an ICP of greater than $50 \mathrm{~mm} \mathrm{Hg}$. The patient's daily fluid balance is featured in Fig. 4. She was found to be hypoadrenal on Day 16. After starting steroid agents, we were able slowly to discontinue her pressor agents. During the first 2 weeks postinjury, she also developed severe ARDS with a fractional inspired oxygen level of $100 \%$, positive end-expiratory pressure levels of $36 \mathrm{~mm}$ $\mathrm{Hg}$, mean airway pressures of $70 \mathrm{~mm} \mathrm{Hg}$, and peak pressures of $90 \mathrm{~mm} \mathrm{Hg}$. She did not develop renal insufficien-

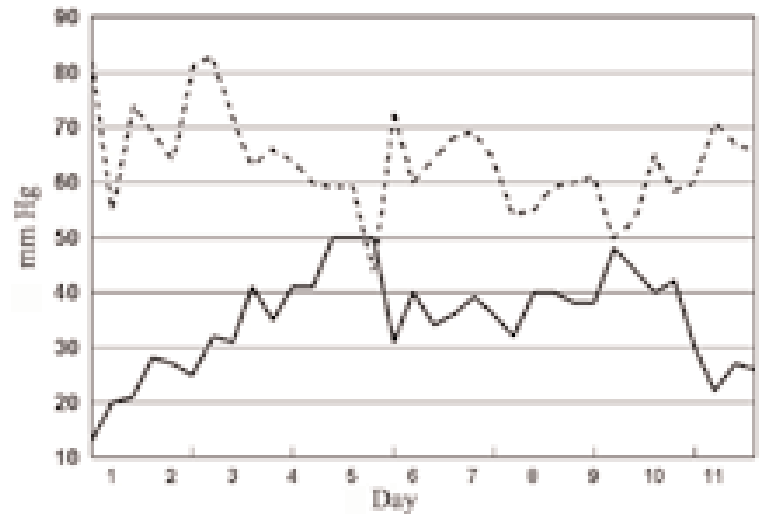

Fig. 2. Case 1. Graph of hourly ICP (solid line) and CPP (dotted line) measurements obtained in the ICU.

cy until after her ICP problems had subsided. The patient was weaned off the ventilator over the course of 2 months and discharged to a rehabilitation facility. At the time of discharge, she was awake and oriented to time, place, and people; able to feed herself and dress her upper body; and was beginning vigorous lower-extremity physical therapy. She was discharged from rehabilitation after 2 months and was assigned a GOS score of 4 .

Case 2. This 49-year-old man was involved in a rollover MVA and sustained injuries that included closed head injury (GCS Score 3 on admission), hemopneumothorax, $\mathrm{T}-7$ burst fracture with spinal cord injury, and multiple lacerations. His initial cranial CT scan was nondiagnostic. He was hemodynamically stable and his initial lactic acid level was 6.4. $\mathrm{mmol} / \mathrm{L}$. His ISS was 36. After the initial diagnostic workup, an ICP monitor was placed and its initial reading was $8 \mathrm{~mm} \mathrm{Hg}$. A Swan-Ganz catheter was placed and resuscitation was ongoing. The patient had a severe pulmonary contusion and manifested symptoms of ARDS within the first 3 days postinjury. He had excellent CPP values until his 3rd postinjury day (Fig. 5), when he manifested hemodynamic instability due to sepsis from a likely combination of pulmonary contusion and aspiration

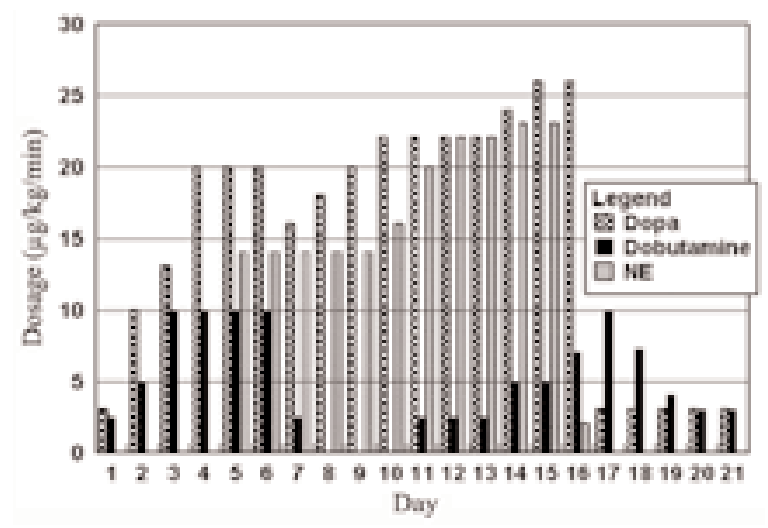

Fig. 3. Case 1. Graph of intravenous dosages of vasopressor agents administered hourly. Day 16 was the time when intravenous steroid agents were begun. 


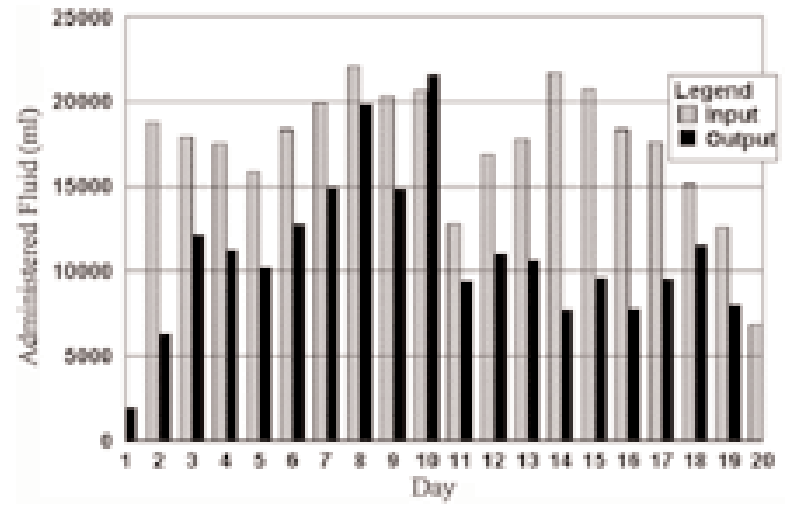

Fig. 4. Case 1. Graph of daily fluid balance. This patient had a large positive fluid balance for the first 2 weeks after injury.

at the time of the accident. At this time, continuous venovenous hemofiltration was initiated because of renal insufficiency, which further exacerbated his hemodynamic problems. This patient then required significant doses of pressor agents for several days (Fig. 6). His ICP remained elevated, and the pressure spikes correlated well with periods of hemodynamic instability. He was weaned off the ventilator with the aid of a tracheostomy and was discharged to a rehabilitation facility. On discharge from rehabilitation, he could feed and dress himself, his memory was intact, and he was able to complete bed-to-chair transfers by himself and was paraplegic from his spine injury. He was assigned a GOS score of 4 .

Case 3. This 39-year-old woman who was involved in a high-speed MVA, was ejected from the vehicle and suffered respiratory arrest at the scene. On admission, she was assigned a GCS Score 3, she was intubated, and her condition was hemodynamically unstable. She had a positive peritoneal lavage and was taken to the operating room for laparotomy after a rapid cranial CT study. The CT scan revealed mild subarachnoid hemorrhage and mild swelling. Her injuries included a massive left diaphragmatic laceration, radius fracture, femur fracture, right posterior knee dislocation, hemopneumothorax, cardiac contusion, severe liver laceration, and nonbleeding spleen

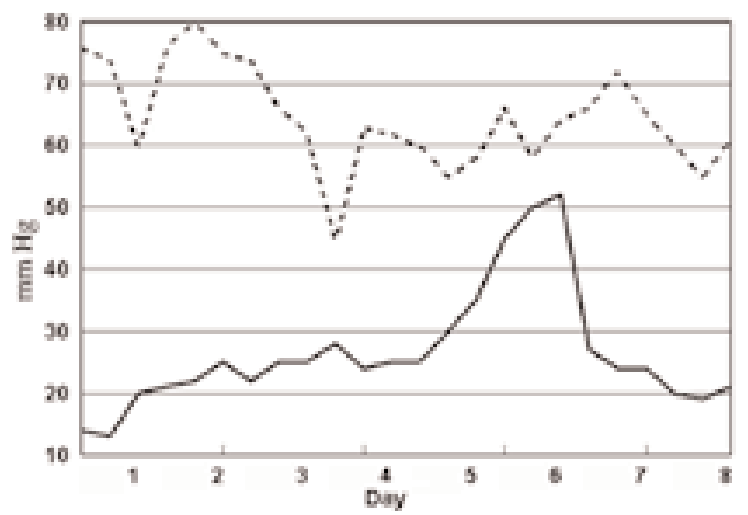

Fig. 5. Case 2. Graph of hourly ICP (solid line) and CPP (dotted line) measurements.

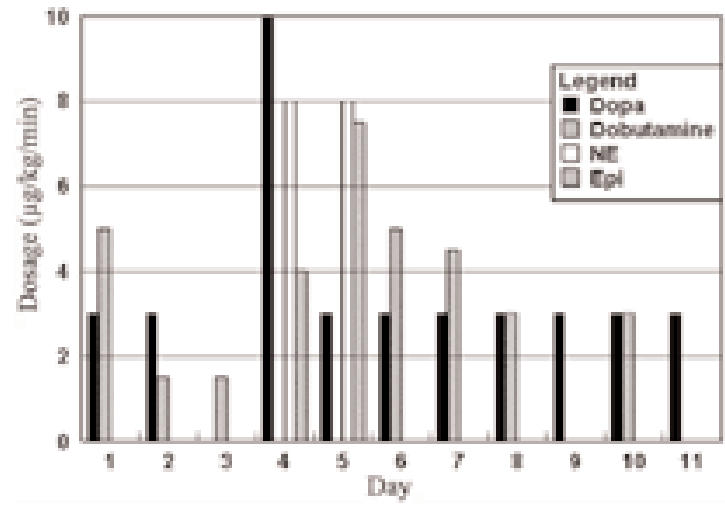

Fig. 6. Case 2. Graph of intravenous vasopressor dosages administered hourly. Epi = epineprine; $\mathrm{NE}=$ norepineprine.

laceration. She received $16 \mathrm{U}$ of blood in the first 24 hours postinjury, her ISS was 57, and her probability of survival was 0.014 . Her initial lactic acid level was $8.2 \mathrm{mmol} / \mathrm{L}$. She had an ICP bolt placed concurrent with a laparotomy and underwent vigorous resuscitation in the first 24 hours postinjury. The patient's ICP and CPP trends are featured in Fig. 7. We were able consistently to keep her CPP values higher than $60 \mathrm{~mm} \mathrm{Hg}$ through the use of fluid resuscitation and pressor agents. She underwent tracheostomy and was weaned off the ventilator over the course of 4 weeks. She was discharged to inpatient rehabilitation, and 4 months after admission she has a GCS score of 15 and she is completing mild housework, dressing herself, and performing all activities of daily living. Her GOS score is 4.

Case 4. This 39-year-old woman was involved in a head-on MVA. Her injuries included a severe head injury (GCS Score 3) with CT scanning evidence of shear injury and subarachnoid hemorrhage, basilar skull fracture, open right eye injury, orbital floor fracture, LaFort II facial fracture, patellar fracture, bilateral pneumothoraces, retroperitoneal hematoma, and bilateral pulmonary contusions. Her ISS was 50 and her initial ICP was $36 \mathrm{~mm} \mathrm{Hg}$. Her ICP and CPP trends are featured in Fig. 8. She required multiple pressor agents and massive fluid resuscitation for

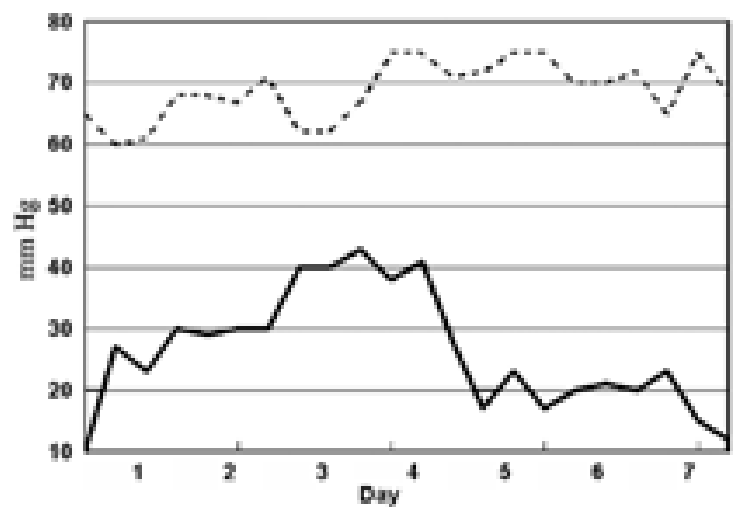

Fig. 7. Case 3. Graph of mean ICP (solid line) for each 8-hour interval and CPP (dotted line). 


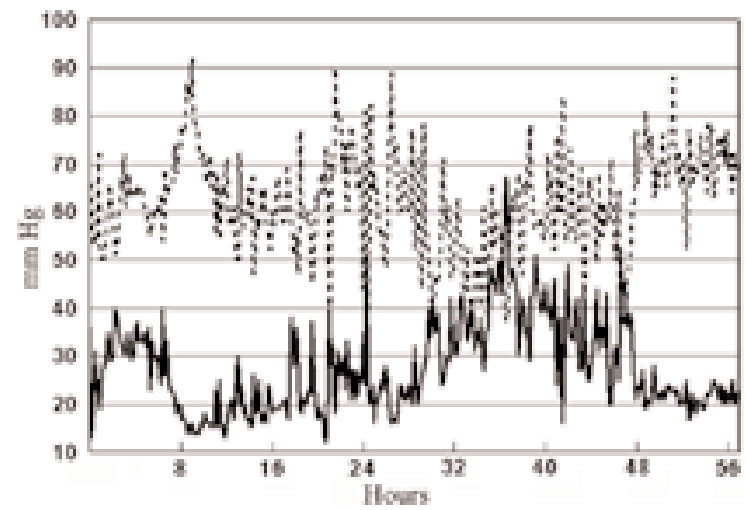

Fig. 8. Case 4. Graph of mean ICP for each 8-hour interval.

maintenance of CPP. Her complications included pneumonia and ARDS requiring nitric oxide treatment as well as oscillating ventilation. Her ICP slowly normalized over the course of 10 days. She was transferred to inpatient rehabilitation after a 30-day hospital stay, awake and oriented, performing activities of daily living, with a GOS score of 4 . Her condition has continually improved as an outpatient.

\section{DISCUSSION}

There has been a paradigm shift in the treatment of severe head injury, that is, from management focused on reducing ICP by any measure to one focused on resuscitation and maintenance of CPP together with ICP control. The work of Rosner and Daughton ${ }^{18}$ and the American Association of Neurological Surgeons guidelines have prompted many centers to reconsider the treatment of severe head injury. At our center, there was constant conflict between the priorities of the trauma intensivists (resuscitation) and the neurosurgeons (dehydration). After intense discussion, it was concluded that patients with multiple injuries were poorly served by under-resuscitation and that there was sufficient data to support CPP-directed therapy as a matter of protocol.

The argument for CPP-directed management is compelling. Increasing evidence demonstrates that cerebral blood flow is very low following TBI and, in fact, may be near the ischemic threshold. ${ }^{4,5}$ It has also been shown in histological analyses of brains in patients who died following TBI that ischemia is very common..$^{9,10}$

In several studies, ${ }^{8,14}$ hypotension has been found to be extremely detrimental to outcome following severe TBI. There are also experimental data indicating that a decline in blood pressure is responsible for sudden increases in ICP (plateau waves) and that these waves can be aborted by increasing systemic blood pressure. ${ }^{16}$ One important finding emerged from our change in treatment strategy: when patients are maintained in a hypovolemic-euvolemic state, hypotensive episodes induced by medications, sedation, and surgery are more common than they would be if the patient were maintained in a euvolemic-hypervolemic state. There is concern that hypertension is detrimental to patients with severe TBI. Bouma and Muizelaar ${ }^{3}$ and Bruce and colleagues ${ }^{6}$ found that, at worst, there is a small increase in ICP, and in many cases ICP decreases, with increases in mean arterial pressure. Thus, it is reasonable to conclude that moderate increases in blood pressure required to elevate CPP should not precipitate severe intracranial hypertension in most patients. We found that as mean arterial pressure increased, ICP decreased, and the linear regression had a distinctively negative slope that was significantly different from zero (Fig. 9).

Rosner and colleagues ${ }^{17-19}$ have studied the maintenance of a CPP greater than $70 \mathrm{~mm} \mathrm{Hg}$ in patients with severe TBI. Their results are excellent, with an overall mortality rate of $21 \%$ in these patients. Fifty-four percent of surviving patients had good recoveries or moderate disabilities. Changaris, et al., ${ }^{7}$ found that all patients with TBI whose CPP decreased below $60 \mathrm{~mm} \mathrm{Hg}$ for more than $33 \%$ of the hourly measurements obtained on the 2nd postinjury day died. We chose a CPP of $65 \mathrm{~mm} \mathrm{Hg}$ as our goal in that our preliminary examination of this population revealed that this level was more often associated with the resolution of systemic lactic acidosis and required moderate pressor therapy. In truth, even this CPP goal was very difficult to attain in these cases despite massive fluid and pressor treatment.

There are very few data in the literature that reveal the outcome in patients with severe intracranial hypertension following blunt TBI. In Rosner and Daughton's study, ${ }^{18}$ the mean ICP was $23 \pm 9.8 \mathrm{~mm} \mathrm{Hg}$, and four patients died of high ICP (32-45 mm Hg). The number of patients with a sustained ICP greater than $40 \mathrm{~mm} \mathrm{Hg}$ and their outcomes were not stated in that study. In our experience, neurosurgeons question the validity of a treatment regimen when ICP values are greater than $35 \mathrm{~mm} \mathrm{Hg}$, placing the emphasis on more aggressive ICP-lowering techniques (dehydration, hyperventilation, and so forth) rather than the aggressive maintenance of CPP. The purpose of this report was to provide a description of our experience in attempting to maintain CPP, even in the presence of severe intracranial hypertension. Data collected by Marmarou and colleagues ${ }^{11}$ from the TCDB revealed that as the percentage of instances in which ICP was greater than $20 \mathrm{~mm} \mathrm{Hg}$ increased, the chance of a favorable outcome decreased precipitously. In the TCDB, patients with se-

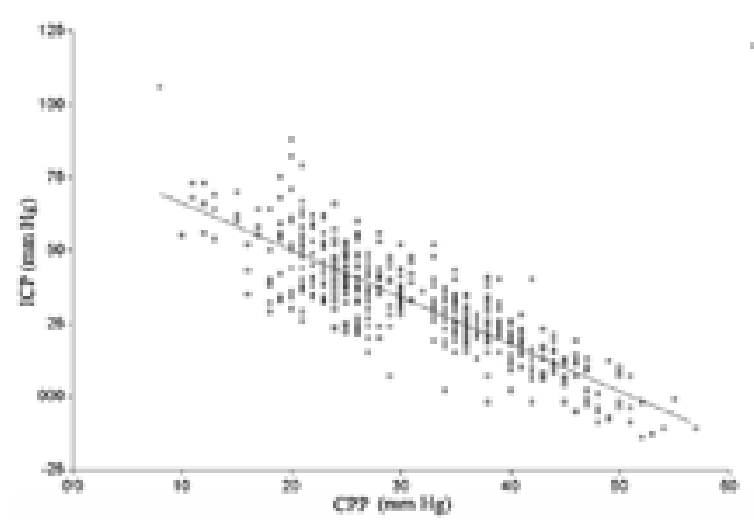

Fig. 9. Linear regression of ICP and CPP for all data points collected in all three patients (number of points 481). Notice the significantly negative slope to the line, indicating that as CPP increas- 
vere cerebral edema demonstrated on $\mathrm{CT}$ scans had a favorable outcome rate of $16.4 \%$, and recovery to independent activity was rare. ${ }^{12}$ Another option for these patients would have been decompressive craniectomy, which is used in our institution. ${ }^{15}$ During discussion, however, the neurosurgeons asserted that other medical issues precluded the use of decompressive craniectomy in these patients given the hemodynamic and respiratory instability in the acute phase of their injuries.

There are several interesting concepts in this preliminary study. Three patients suffered from severe multiple traumas and associated complications that affected their neurotrauma care. In all three, precipitous rises in ICP were preceded by reductions in CPP. Routine critical care issues (sepsis, sedation, turning, and so forth) caused these reductions. Nonetheless, we believe that CPP must be scrupulously maintained above $60 \mathrm{~mm} \mathrm{Hg}$ in all patients with severe TBI for at least the first 96 hours. We were not always successful in maintaining CPP because of nursing and resident inexperience with the protocol; inadequate diligence and aggressiveness; the need for "road trips" because all drugs and fluids present in the ICU were not readily available; and complications such as sepsis, renal failure, and ARDS. As a result, our nursing and physician practices have changed. Trends are more closely examined, road trips are avoided, and pressors are at the patient's bedside at all times if needed. After treating the patients in Cases 1 and 2, we were more attuned to tighter CPP control, and ICP fluctuations were less severe, as demonstrated by the ICP/CPP tracings obtained in the patient in Case 3. When ICP is in the range of 40 to $50 \mathrm{~mm}$ $\mathrm{Hg}$, large doses of pressors are often needed for control of CPP. We found phenylephrine very useful, but the patient must be euvolemic and adequately fluid-resuscitated as a first step toward CPP elevation before pressors are administered. A Swan-Ganz catheter is almost mandatory in these patients to have a clear idea of the fluid status. Adult respiratory distress syndrome was severe in all of these patients. Given that high positive end-expiratory pressure can mask hypovolemia in some cases, more advanced hemodynamic monitoring techniques (oximetric or Ref-Ox catheters) may be required.

\section{CONCLUSIONS}

Although the data in this paper in no way represents a controlled experiment, we believe that it is important to report the encouraging outcomes in these three patients with malignant posttraumatic cerebral edema. We assert that intense management of CPP and aggressive resuscitation can maximize outcome in this group of patients with high risk of death. The reader must bear in mind that aggressive CPP management may require a significant change in nursing and physician practices in the critical care setting and that there is likely to be initial variability in CPP control. Before the institution of this protocol, we did not have one survivor with a sustained ICP greater than $40 \mathrm{~mm} \mathrm{Hg}$. Thus, the four survivors with favorable outcomes have led us to believe that CPP-directed management is essential and can be effective when high ICP is present. We recognize the limited nature of this study, but believe that the data presented herein warrant further investigation.

\section{References}

1. Baker SP, O’Neill B, Haddon W Jr, et al: The injury severity score: a method for describing patients with multiple injuries and evaluating emergency care. J Trauma 14:187-196, 1974

2. Becker DP, Miller JD, Ward JD, et al: The outcome from severe head injury with early diagnosis and intensive management. J Neurosurg 47:491-502, 1977

3. Bouma GJ, Muizelaar JP: Relationship between cardiac output and cerebral blood flow in patients with intact and with impaired autoregulation. J Neurosurg 73:368-374, 1990

4. Bouma GJ, Muizelaar JP, Choi SC, et al: Cerebral circulation and metabolism after severe traumatic brain injury: the elusive role of ischemia. J Neurosurg 75:685-693, 1991

5. Bouma GJ, Muizelaar JP, Stringer WA, et al: Ultra-early evaluation of regional cerebral blood flow in severely head-injured patients using xenon-enhanced computerized tomography. J Neurosurg 77:360-368, 1992

6. Bruce DA, Langfitt TW, Miller JD, et al: Regional cerebral blood flow, intracranial pressure, and brain metabolism in comatose patients. J Neurosurg 38:131-144, 1973

7. Changaris DG, McGraw CP, Richardson JD: Correlation of cerebral perfusion pressure and Glasgow Coma Scale to outcome. J Trauma 27:1007-1013, 1987

8. Chesnut RM, Marshall LF, Klauber MR, et al: The role of secondary brain injury in determining outcome from severe head injury. J Trauma 34:216-222, 1993

9. Graham DI, Ford I, Adams JH, et al: Ischaemic brain damage is still common in fatal non-missile head injury. J Neurol Neurosurg Psychiatry 52:346-350, 1989

10. Graham DI, Lawrence AE, Adams JH, et al: Brain damage in fatal non-missile head injury without high intracranial pressure. J Clin Pathol 41:34-37, 1988

11. Marmarou A, Anderson RL, Ward JD, et al: Impact of ICP instability and hypotension on outcome in patients with severe head trauma. J Neurosurg 75 (Suppl):S59-S66, 1991

12. Marshall LF, Marshall SB, Klauber MR, et al: The diagnosis of head injury requires a classification based on computed axial tomography. J Neurotrauma 9 (Suppl 1):S287-S292, 1992

13. Muizelaar JP, Marmarou A, Ward JD, et al: Adverse effects of prolonged hyperventilation in patients with severe head injury: a randomized clinical trial. J Neurosurg 75:731-739, 1991

14. Pietropaoli JA, Rogers FB, Shackford SR, et al: The deleterious effects of intraoperative hypotension on outcome in patients with severe head injuries. J Trauma 33:403-407, 1992

15. Polin RS, Shaffrey ME, Bogaev CA, et al: Decompressive bifrontal craniectomy in the treatment of severe refractory posttraumatic cerebral edema. Neurosurgery 41:84-92, 1997

16. Rosner MJ, Becker DP: Origin and evolution of plateau waves. Experimental observations and a theoretical model. J Neurosurg 60:312-324, 1984

17. Rosner MJ, Coley IB: Cerebral perfusion pressure, intracranial pressure, and head elevation. J Neurosurg 65:636-641, 1986

18. Rosner MJ, Daughton S: Cerebral perfusion pressure management in head injury. J Trauma 30:933-940, 1990

19. Rosner MJ, Rosner SD, Johnson AH: Cerebral perfusion pressure: management protocol and clinical results. J Neurosurg 83:949-962, 1995

20. Young JS, Meredith JW: Does oxygen delivery-directed resuscitation worsen outcome of head injured patients with multisystem injuries? Am Surg 61:419-423, 1995

\footnotetext{
Manuscript received November 6, 2003.

Accepted in final form November 12, 2003.

Address reprint requests to: Jeffrey S. Young, M.D., F.A.C.S., P. O. Box 800709, University of Virginia Health System, Charlottesville, Virginia 22906-0709. email: JSY2B@ hscmail.mcc.virginia.edu.
} 